\title{
FRONTERAS Y ESPACIOS FRONTERIZOS EN ÁFRICA CENTRAL*
}

\author{
Por \\ Karine Bennafla**
}

\begin{abstract}
RESUMEN
Los espacios fronterizos en África central experimentan desde hace quince años una efervescencia de actividades comerciales, en un contexto general de un callejón sin salida económico y político. Esas zonas transfronterizas emergentes se ven atravesadas a escalas inferiores o superiores por otras fronteras (monetarias, culturales, políticas) que se superponen con los límites Estatales, lo que hace complejo el funcionamiento de esos espacios. Dichos espacios son estructurados en función de mercados y de ejẹs de comunicación que los ponen en contacto con las ciudades interiores, consumidoras y proveedores de mercancías. Los problemas de seguridad, de control policiaco, el mantenimiento desigual de las carreteras explican el carácter movedizo de los itinerarios hacia la frontera, la duración efímera de los mercados fronterizos y la ausencia de inversiones inmobiliarias sobre los confines. Los espacios transfronterizos son, al fin, construcciones fugaces, pero estrechamente conectadas con los centros urbanos del interior, consolidando el marco existente de los territorios nacionales.
\end{abstract}

\begin{abstract}
The frontier spaces of Central Africa have for the last fifteen years been experiencing bubbling commercial activity, within a general context of an economic and political blind alley. These emerging transborder zones are crisscrossed on a lasser or greater scale by other boundaries (monetary, cultural, political) that are superimposed with the national perimeters. For this reason, these spaces are very complex in the way they function. They are structured with regard to markets and the avenues of communication that put them in contact with cities in the interior, comsumers and providers of merchandise. Problems of security, of police control, of uneven maintenance of roadways explain the constant movement of real restate investments on the borders. The transborder spaces are, when all is said and done, fleeting structures but closely connected with the interior urban centers, consolidating framework of the national territories.
\end{abstract}

* Traducido del francés por Danielle Wynants Raeymaekers.

** Agregada temporaria de enseñanza y de investigación en la Universidad de Cergy-Pontoise. 


\section{INTRODUCCIÓN}

Cerca de 40 años después de la independencia, el resumen socioeconómico de la mayoría de los Estados africanos ya no es muy brillante. Si nos referimos a las estadísticas del Banco Mundial, la participación del continente negro en el comercio internacional se coloca por abajo del 3\% al principio de los años noventa. Esa marginación económica refleja el estado de deterioro de las economías nacionales africanas. Los seis países de África central que se estudian aquí, principalmente ${ }^{1}$, (Nigeria, Camerún, Chad, Centroafrica, Gabón, Guinea Ecuatorial) no son la excepción, incluso si hay matices que pueden ser operados según el caso: todos están, desde los años ochenta, sumidos en una recesión económica duradera y tuvieron que tener terapias de choque o como dicen algunos africanos, los choques sin terapia de los Planos de Ajustes Estructurales (PAS) elaborados por los expertos en liberalismo del Banco Mundial y del Fondo Monetario Internacional (FMI). Las poblaciones africanas pagaron el precio elevado de las medidas de austeridad destinadas a volver a equilibrar el presupuesto del Estado y cuyos corolarios se conjugan sicmpre siguiendo la misma letanía: disminución del poder adquisitivo de los habitantes, degradación de los servicios de salud y de educación, pobreza acrecentada de las clases sociales bajas y medias en la ciudad, aumento de la delincuencia, multiplicación de actividades de supervivencia y de tráficos ilegales.

Esa coyuntura socioeconómica desfavorable se conjuga con un medio ambiente político igualmente crítico ya que está marcado por una crisis del Estado y un disfuncionamiento de las estructuras estatales de mando. "Política del vientre" (J.F. Bayard, 1989), "privatización del Estado" (Bayard, Ellis e Hibou, 1997), "superposición de las posiciones del poder y de acumulación" (Bayard, Ellis e Hibou, 1997), son algunas de las perífrasis que abundan para nombrar la corrupción generalizada a todos los niveles, que acabaron por vaciar el Estado de su sustancia y por descreditarlo como administrador, como gestor y como autoridad normativa.

En un contexto tal de bancarrota estatal, de callejón sin salida económica y de incertidumbre en cuanto al mañana, asistimos a la puesta en marcha de nuevos procesos de recomposición territorial, donde el más espectacular es la cmergencia de zonas transfronterizas animadas por flujos comerciales transversales. La particularidad de los espacios fronterizos africanos es, en efecto, apoyarse casi exclusivamente sobre actividades

1 Este artículo se utilizó información rebabada en trabajo de campo de un proyecto de tesis. 
de intercambio (más o menos controladas) polarizadas por aldeas-mercados. Esa reconfiguración de los espacios de intercambio para el provecho de los márgenes fronterizos tiende a demostrar que los Estados, empobrecidos y a menudo inestables, han perdido el mando de su territorio y se ven forzados a dejar que sus periferias se administren solas. Todo sucede como si las formas antiguas de organización territorial se hubieran vuelto caducas, como si la disposición piramidal del Estado moderno administrado en la cima por la capital fuera de ahora en adelante inoperante. ¿La efervescencia de orden comercial que afecta desde hace diez años ciertas zonas fronterizas representa, por lo tanto, una amenaza para el Estado y los procesos de construcción y de integración nacionales?

El tema de este artículo es cuestionar sobre la noción de frontera y las especificidades de los espacios fronterizos en África, en el sur del Sahara, teniendo como punto de salida cuatro ejemplos colocados en África central, en medios biogeográficos muy diferentes: el espacio fronterizo Gabón-Guinea Ecuatorial-Camerún se inscribe en regiones subpobladas de la selva ecuatorial; la zona Chad-Libia se extiende en pleno desierto del Sahara; las otras dos regiones comprenden para una, el norte de Nigeria, el norte de Camerún y el Chad, y para la otra, Camenún, Chad y Centroafrica; corresponden respectivamente a una zona sahelo-sudanesa esteparia y a una zona más húmeda de sabana (figura 1). Esos espacios tienen en común ser nuevos lugares de animación y de movimientos. ¿Cómo es que esas regiones periféricas en general abandonadas por la red de comunicación moderna y por la mayoría de los proyectos de desarrollo se han vuelto lugares de sinergia concentrando hombres y actividades? ¿cuáles son las consecuencias de esa actividad fronteriza en el plan político territorial?

\section{UNA MULTITUD DE FRONTERAS UBICADAS EN DIFERENTES ESCALAS}

Los espacios fronterizos no podrían resumirse en una sencilla intersección de fronteras estatales. Están cercados y atravezados por varios tipos de fronteras, lo que hace más compleja la lectura de su funcionamiento por el hecho del enrredo de reglas y de leyes muy diversas, y a veces contradictorias. A diferentes escalas, las fronteras se suceden envolviendo de esa manera espacios encajados uno en el otro cuya región transfronteriza no es más que uno de los estratos. En medio de esas fronteras que se enciman una sobre otra, podemos distinguir las fronteras supranacionales de la zona franca y de la Unión Aduanera y Económica de los Estados de África Central (UDEAC), las de los territorios nacionales, y por fin, a gran escala, las fronteras internas que pueden ser, por ejemplo, de orden cultural o 
FRONTERAS Y ESPACIOS FRONTERIZOS EN ÁFRICA CENTRAL

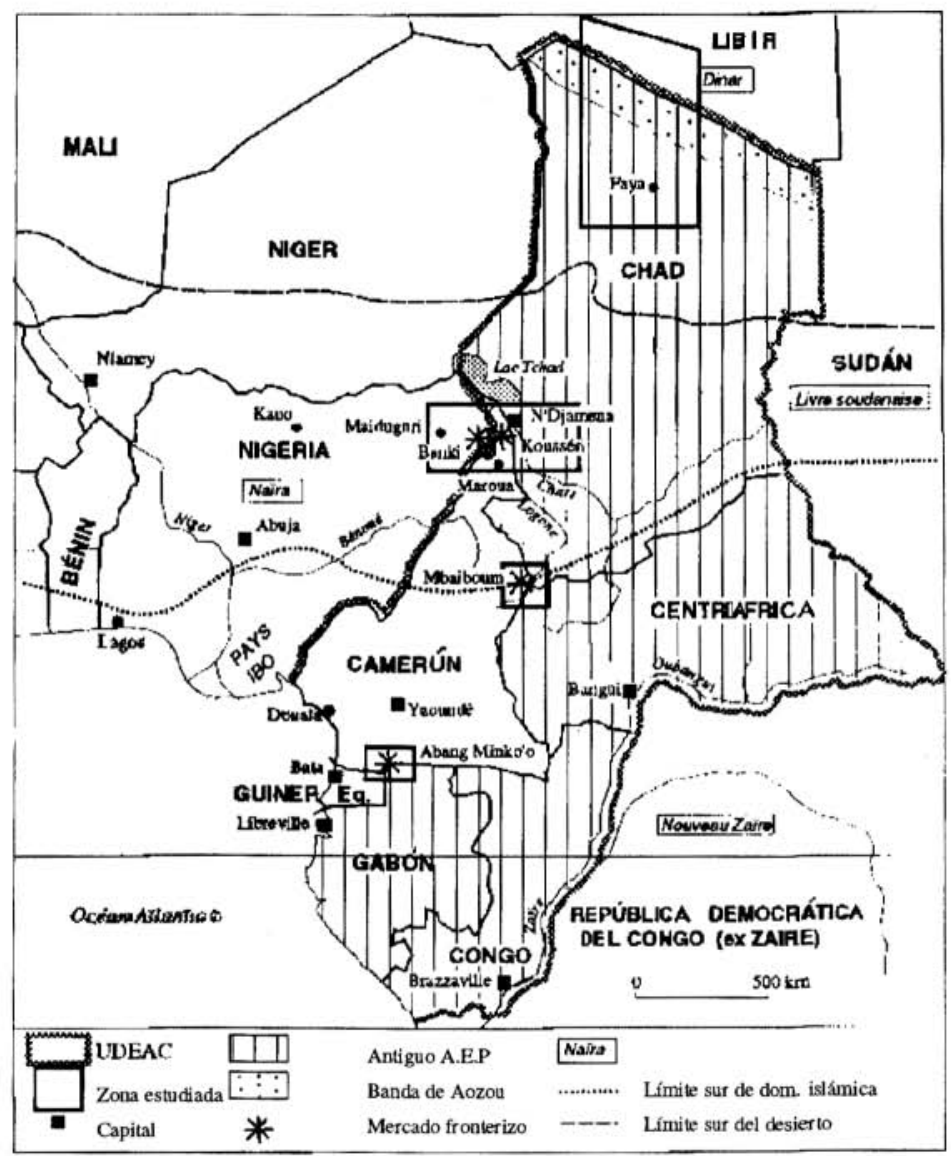

Figura 1. Ubicación de las zonas transfronterizas estudiadas en África central.

Fuente: Atlas Jeune Afrique. 
étnico. Cada una de esas fronteras delimita, a su nivel, un conjunto provisto de códigos y de reglamentos propios cuyo espacio transfronterizo hace eco de ellos.

\section{Las fronteras supranacionales}

\section{Las fronteras monetarias de la zona franca}

A escala pequeña, las fronteras de la zona franca (figura 2) encierran un vasto dominio en África occidental y central, de las costas del Atlántico desde los contrafuertes de Ouaddaï, del desierto del Sahara hasta las profundidades silvestres de la cuenca del congo. En el interior de ese conjunto geográfico (construido alrededor de las antiguas colonias francesas) circula el franco CFA cuya particularidad es la de gozar del beneficio de un libre cambio y una paridad fija frente al franco francés $(1 \mathrm{FF}=100 \mathrm{~F}$ CFA desde 1994). Esa convertibilidad y esa estabilidad hacen del franco CFA una moneda fuerte y buscada en una región caracterizada por la importancia de las monedas flotantes y de la inflación (incluso hiperinflación para Zaire). La diferencia entre el franco CFA y las monedas de los países vecinos constituye uno de los factores principales de los intercambios transfronterizos. La frontera entre el franco CFA y la moneda nigeriana, el naira, es sin duda alguna la que tiene la más fuerte influencia en la subregión; mercados se implantaron así a lo largo de la línea fronteriza nigeriana. La no convertibilidad del naira y su continua depreciación frente al franco CFA desde el principio de los años ochenta contribuyen al derrame de los productos manufacturados nigerianos hacia los países de África central miembros de la zona franca, Camerún funcionando como un vasto espacio de tránsito y de redistribución. El cambio naira-franco CFA favorable a este último hace que los precios de las mercancías provenientes de Nigeria sean muy atractivos para los compradores que tienen moneda CFA. Por su parte, los hombres de negocios nigerianos son cuidadosos de cambiar sus nairas incambiables por una divisa tal como lo es el CFA. De allí, el flujo (a menudo en contrabando) hacia los países de África central de productos de consumo corriente de origen nigeriano (telas, vajillas, calzado, material de sonido $h i-f i$, carros, azúcar, cigarros, etcétera). Debido a que evaden a menudo los aranceles y la aduana, esos productos nigerianos compiten severamente con las empresas locales de los países de la zona franca. La devaluación del franco CFA de 50\% producida en enero de 1994 , se resintió duramente en el nivel de los mercados fronterizos, conllevando durante largos meses una disminución neta dc ias transacciones comerciales y una baja de actividades, ésta por el nuevo aumento del costo de 
los productos nigerianos y por la baja del poder adquisitivo de la poblacion en los países de la zona franca. La devaluación se tradujo, por una modificación de la paridad franco CFA-naira, en provecho de este último; el cambio de 1,000 CFA que estaba sobre el mercado de Garoua (norte de Camerún) de 135 nairas a la víspera de la devaluación, regresó en mayo de 1994 a 70 nairas; sobre el mercado de Camerún, la venta de los productos de fabricación camerunesa fue de alguna manera favorecida frente a las mercancías nigerianas, mientras que las exportaciones del ganado del Chad y Centroafrica hacia Nigeria aumentaron sensiblemente en 1994. Sin embargo, un año después de la devaluación, las caídas repetidas del naira devolvió su paridad con respecto al franco CFA a los niveles de antes, anulando el efecto de devaluación.

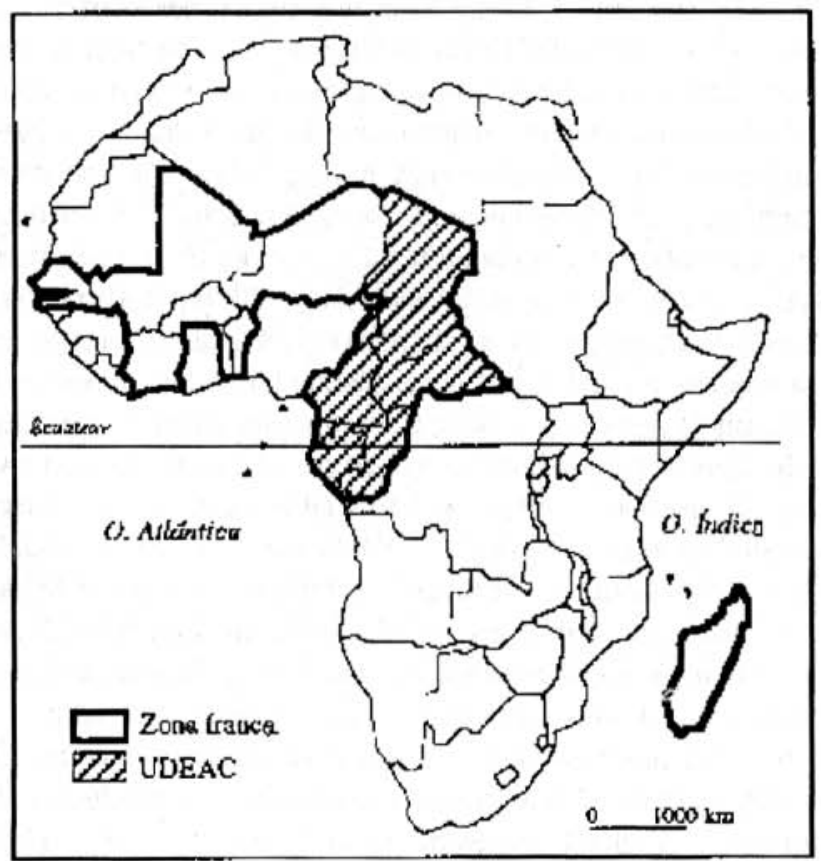

Figura 2. Zona franca y UDEAC. 
En el interior de la zona franca, existe una frontera entre África central y África del oeste: el banco de los Estados de África del oeste emite el franco de la "comunidad financiera africana" (CFA) que difiere ${ }^{2}$ del franco de la "cooperación financiera de África central (CFA), emitido por los bancos de los estados de África central. La zona de emisión del franco CFA de África central corresponde a la zona de la unión aduanera y económica de los estados de África central.

\section{Las fronteras de la zona UDEAC}

A una escala media, las fronteras del conjunto comunitario UDEAC (ver figura 2) también tienen repercusiones sobre el desarrollo del comercio fronterizo. Nacida en 1964, la UDEAC agrupa seis países (Camerún, Congo, Chad, Centroafrica, Gabón y Guinea Ecuatorial) y corresponde, de hecho, a "na reminiscencia de la antigua África ecuatorial francesa (AEF) que agrupaba a Chad, Centroafrica, el Congo y Gabón actuales. A ese duro núcleo de la UDEAC se agrega en 1985 Guinea Ecuatorial, antigua colonia española, desde entonces miembro de la zona franca. La UDEAC que preconizaba en sus textos fundadores la libre circulación de los hombres y de las mercancías entre los países miembros, se quedó como una concha vacía, carente de sustancia y de realidad concreta hasta el final de los años ochenta. Al principio de los años noventa, los responsables de la Secretaría de la Cooperación Francesa y de la Unión Europea, inspirados por los modelos de integración regional europeo (Unión Europea) y americana (ALENA) se dieron a la tarea de volver a hechar andar esa construcción artificial. Se lanzó un vasto programa de reformas en 1991, incluyendo una política de transporte, así como una tabla fiscal y aduanera para facilitar el tránsito interestatal. La aplicación desde 1994 de una reforma fiscal y aduanera destinada a implantar una tasa común a la entrada de la zona sobre las mercancías proveniendo de fuera de la UDEAC y a suprimir progresivamente los impuestos y derechos de aduana entre los Estados miembros, ha empezado a afectar los intercambios fronterizos. Así pues, las mercancías nigerianas que transitan a Mbaiboum (mercado camerunés situado en la intersección de las fronteras camerunesas, chadianas, y de Centroafrica) hacia Centroafrica pagan de ahora en adelante sus derechos de aduana a la garita fronteriza centroafricana vecina, con tasas más elevadas que las de los productos cameruneses.

2 Desde septiembre de 1993, los billetes emitidos por los dos bancos centrales ya no son libremente cambiables de una comunidad monetaria a otra. 


\section{Las fronteras nacionales: una génesis reciente, un origen colonial}

Las fronteras del Estado sin duda son aquellas cuyo papel impone más al espíritu en la organización y la estructura interna de los espacios transfronterizos por las diferencias políticas, económicas y jurisdiccionales que generan.

Las fronteras nacionales de África central son el reflejo de una rejilla espacial heredada de las competencias coloniales; delimitan entidades estatales que han sido creadas completamente por los europeos al final del siglo XIX, cuando el continente fue dividido por tratados firmados rápidamente sobre la base de mapas inciertos. La delimitación de las zonas de influencia extranjera en la región central de África tuvo lugar entre la conferencia de Berlín (noviembre de 1884 y febrero de 1885) y la primera Guerra Mundial, al término de una verdadera carrera para lograr territorio. En el curso de esa carrera, las potencias colonizadoras europeas, sobre todo Francia (presente en AEF) España (en Guinea Ecuatorial), Alemania (en Camerún hasta 1916) e Inglaterra (en Nigeria y Libia) se pelearon el pastel africano. Sobreimpuestas en un continente negro que en esa época estaba poco o mal conocido pero que los europeos veían como una "tierra vacante y sin dueño", las fronteras africanas son, según la terminología de M. Foucher, fronteras exógenas. Remiten ya sea a antiguas líneas de reparto entre dos imperios coloniales, o a viejos límites intraimperiales.

Trazada en 1894, la frontera oriental de Camerún corresponde al primer caso (un origen interimperial) ya que inicialmente seperaba Camerún, bajo protectorado alemán, del Chad y de Oubangui-Chari (hoy Centroafrica) los dos incorporados a África Ecuatorial francés (AEF). La frontera chadocentroafricana muestra el caso de una frontera de origen intraimperial ya que se trata de una delimitación interna a la AEF.

Las fronteras contemporáneas de África central han sido fijadas pues entre el final del siglo XIX y el entre dos guerras, con la excepción notable del límite occidental de Camerún que se debe a la descolonización. Después de la derrota alemana en la primera guerra mundial, Camerún se había vuelto en 1918 un territorio bajo mandato de la Sociedad de las Naciones (SDN): los tres cuartos del éste fueron colocados bajo tutela francesa, mientras que la fracción del oeste pasó bajo tutela de los británicos y fue administrada como parte integrante de su colonia nigeriana. El día siguiente del referendum de 1961 organizado por las autoridades británicas, la población anglosajona de Camerún británico optó por depender de Nigeria, la del suroeste escogió la reunificación a Camerún francófono nuevamente independiente. 
Frecuentemente apoyado sobre líneas rectas "astronómicas" (por ejemplo: entre Chad y Libia), sobre referencias hidrográficas (como el lecho de Logone entre Camerún y Chad) o topográficas (como la línea de cimas del Monte Ngaoui en el macizo de Yadé entre Camerún y Centroafrica) el trazo de las fronteras africanas atestigua la imposición brutal y arbitraria de un modelo europeo de Estado nación, circunscrito de manera lineal, a civilizaciones extranjeras. La configuración territorial de Guinea Ecuatorial, un rectángulo trazado con el cordel, ofrece el ejemplo caricaturesco de la "frontera de cancillería" elaborada en reuniones secretas, sin tomar en cuenta las realidades del terreno. Después de la independencia, las élites africanas en el poder tuvieron cada una que componer un territorio bordeado de fronteras lineales, un territorio-fósil correspondiente a una construcción geopolítica datada.

Esas fronteras heredadas de la colonización parecen haber estado muy interiorizadas por las poblaciones y no se piensan cambiar hoy en día. Es cierto que no faltan los puntos litigiosos como lo ilustra el aumento de tensión, desde 1994, entre Nigeria y Camerún acerca de la península de Bakassi o el conflicto de la banda de Aozou que contrapone a Libia y Chad. Sin embargo, esas diferencias enfrentan sobre todo a los gobiernos y movilizan poco a la población a pesar de los intentos de las autoridades para utilizarlos en las tensiones políticas y socioeconómicas internas. Detrás de los discursos oficiales centrados sobre los temas de seguridad y del honor patriótico se esconden a menudo lo que esté en juego en el orden geoestratégico o económico (por ejemplo, el subsuelo de la península de Bakassi tiene importantes yacimientos de potróleo y de gas off-shore). $\mathrm{Si}$ esas tensiones en las fronteras dan un golpe al "principio de impermeabilidad de las fronteras heredadas de la colonización" contenido en la carta magna de la Organización de la Unidad Africana (OUA), el trazado de las fronteras nacionales globalmente no se altera. En ese sentido, la reestructuración actual de los espacios fronterizos se elaboran "en frío".

En el interior de las fronteras nacionales, las políticas llevadas desde la independencia y la trayectoria específica seguida por los diferentes países de África central crearon diferencias sensibles en los ámbitos demográfico, político, agrícola e industrial.

Sobre el plan demográfico, las desigualdades son muy asombrosas:con 100000000 de habitantes, Nigeria constituye el Estado-gigante de la subregión de África central (figura 3), esta última corresponde a un área de subpoblación (la densidad promedio es de $8 \mathrm{hab} / \mathrm{km}^{2}$ ). Con $14000000 \mathrm{de}$ habitantes en 1997, Camerún sobresale de sus vecinos de Gabón y de Guinea Ecuatorial cuyas poblaciones respectivas se estiman de 1400000 habitantes. Menos flagrante, el contraste de población entre, por una parte 


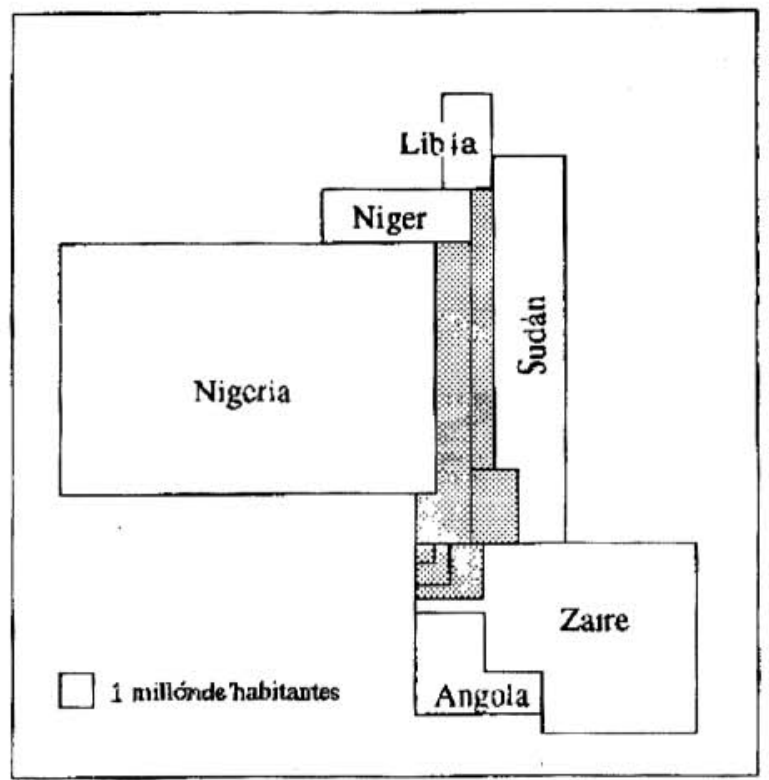

\section{Figura 3. Población de África central.}

Camerún y por otra parte, RCA y Chad ( 3000000 y 6000000 de habitantes) es notable. A escala regional, podemos notar igualmente que a excepcion de la frontera de Chad y Libia, las regiones fronterizas que están en contacto, tienen en común presentar densidades promedias de población que son relativamente elevadas en la escala de África central y que, en la escala nacional, hacen de cada zona nacional fronteriza una de las regiones más pobladas de su país.

De ambos lados de las fronteras, la diferencia económica es patente; padeciendo de una indiferencia industrial y de la desventaja del enclave, Chad y Centroafrica son países que requieren productos de consumo corriente. Por el contrario Nigeria y Camerún tienen el beneficio del acceso al mar y de una red industrial relativamente poderosa y diversificada (sobre todo Nigeria); así pues, son proveedores de productos manufacturados. Cuando los indicadores socioeconómicos de Guinea Ecuatorial muestran un país desangrado, con una economía de desastre, "el emirato" de Gabón puede ufanarse de poseer el ingreso más alto de la África negra gracias a su renta petrolera, minera (manganesio, uranio) y selvática. Aunque no 
están excluidos los problemas económicos, Gabón conserva aún una imagen de "el dorado", ciertamente un poco empañada. La mentalidad y los comportamientos rentistas de muchos gaboneses, heredados de los años fastos 1974-1985 colocados bajo el signo del dinero fácil, ${ }^{3}$ así como el privilegio dado a las inversiones inmobiliarias contribuyen a explicar las pocas preocupaciones de ese país en materia de desarrollo rural y de productos alimenticios para la población local.

En el plano político, los países de África central se hacen notar por el disfuncionamiento de las instancias estatales y una disminución de su soberanía. Esa debilidad de los Estados se debe en gran medida a su génesis reciente, que explica el difícil arraigo de los conceptos importados de nación y ciudadanía. Poder fundado sobre una base etnoregional, prácticas de desvío y uso de la fuerza armada son el destino común de esos Estados frágiles y a menudo inestables. Pero diferencias sensibles entre los estados son percibidas por las poblaciones, en particular en materia de seguridad, de violencia política o de trabas administrativas. Herido por 20 años de guerra civil, Chad presenta un Estado vacante y deteriorado, dirigido por Idriss Déby que tomó el poder por la fuerza en 1990 antes de ser legitimado por las elecciones en 1996. La omnipresencia de hombres armados (militares, ex soldados, ${ }^{4}$ fuerzas del orden) crea un clima de inseguridad y de violencia. Penosamente vuelto al poder gracias a los soldados franceses en junio de 1996, después de una serie de motines militares, el presidente centroafricano Patassé se mantiene vacilante a la cabeza del Estado. En Camerún y en Gabón, los presidentes Biya y Bongo, respectivamente en el lugar desde 1983 y 1967, más o menos se mantienen en el poder a base de elecciones fraudulentas y de una represión más o menos severa de la oposición. Todos ellos están preocupados por guardar una fachada de democracia, no es así para los dirigentes de Nigeria (Sani Abacha) y de Guinea Ecuatorial (Théodoro Obiang Nguema) que instauraron regímenes militares autoritarios.

\section{Las fronteras internas}

A gran escala, fronteras internas se intercalan en el interior de los territorios nacionales; pueden ser de orden cultural, ecológico, político, etcétera. El

\footnotetext{
3 Y eso, gracias a los recursos que provienen de los productos energéticos y mineros.

4 En el marco del proceso de democratización empezado en 1990, el gobierno de Chad redujo los efectivos del ejército: de 40000 en 1990, el número de soldados pasó a 28000 en 1995. Muchos soldados, dados de baja han utilizado su "pri...ia de salida" para comprar un arma y unirse a las bandas.
} 
aeropuerto de N'djamena, capital de Chad, ofrece el ejemplo de una frontera interna en el seno de la zona Chad-Camerún-Nigeria. Aquí los caminos de terracería, los edificios aeroportuarios, las oficinas de policía y de aduana marcan materialmente la frontera. Otra ilustración de frontera interna son las fronteras de la jefatura tradicional autóctona (peule) de Rey Bouba en Camerún (figura 4), que es una de las más poderosas de África. Esa jefatura musulmana, en el lugar desde el fin del siglo XVIII, engloba una superficie comparable a Suiza y forma verdaderamente un Estado dentro del estado en Camerún a causa de la autonomía político-administrativa y judicial de la cual tiene los beneficios de hecho. El territorio de Rey Bouba está materialmente delimitado con barreras móviles de madera o de fierro, instaladas sobre las principales pistas de terracería y vigiladas por servidores a las órdenes del jefe "peul". Usando su posición de señor y dueño, Bouba puso en funcionamiento un sistema de control y de impuestos en la región fronteriza del noreste de Camerún; es así un verdadero gerente y beneficiario del mercado de Mbaiboum. Por ejemplo, los conductores de los camiones de carga que vienen a proveer el mercado tienen que pagar un impuesto arbitrario en cada una de las barreras construidas sobre las carreteras de la jefatura si quieren continuar su camino. En fin, en Mbaiboum, comerciantes, tenderos y prostitutas son objeto de una extorsión de parte de los hombres de confianza del jefe "peul" que no dudan en servirse de la mercancía, en pegar con un bastón, en encarcelar o en matar en caso de que los conductores de camiones de carga no paguen.

Además de ese tipo de fronteras internas de orden político, también podemos apreciar el paso de fronteras lingüísticas, religiosas o étnicas a través de espacios fronterizos. Con respecto a ello, África central es burdamente dividida entre una frontera norte donde predominan los musulmanes y la parte sur, más animista o cristiana (ver figura 1). Esa dicotomía cultural se representa en los mercados fronterizos, a nivel de paisaje y de reparto del comercio. Los edificios religiosos, iglesias y mezquitas, financiadas por los comerciantes en cuanto sus negocios tienen éxito, marcan en efecto los mercados y son más notables debido a que son quizá de los únicos edificios construidos en toda forma, con ladrillos y cemento. Situado sobre la línea de división religiosa, pero en Camerún septentrional, dominado económicamente y políticamente por las élites musulmanas, el mercado de Mbaiboum está dividido entre comerciantes ibos cristianos (originarios de sureste de Nigeria), y de tenderos musulmanes (Peulos del norte de Camerún, árabes de Chad, Haoussas del norte de Nigeria). Parece conveniente recalcar que los comerciantes cameruneses originarios del sur de Camerún, en particular los Bamilékés, hombres de negocios afamados, 


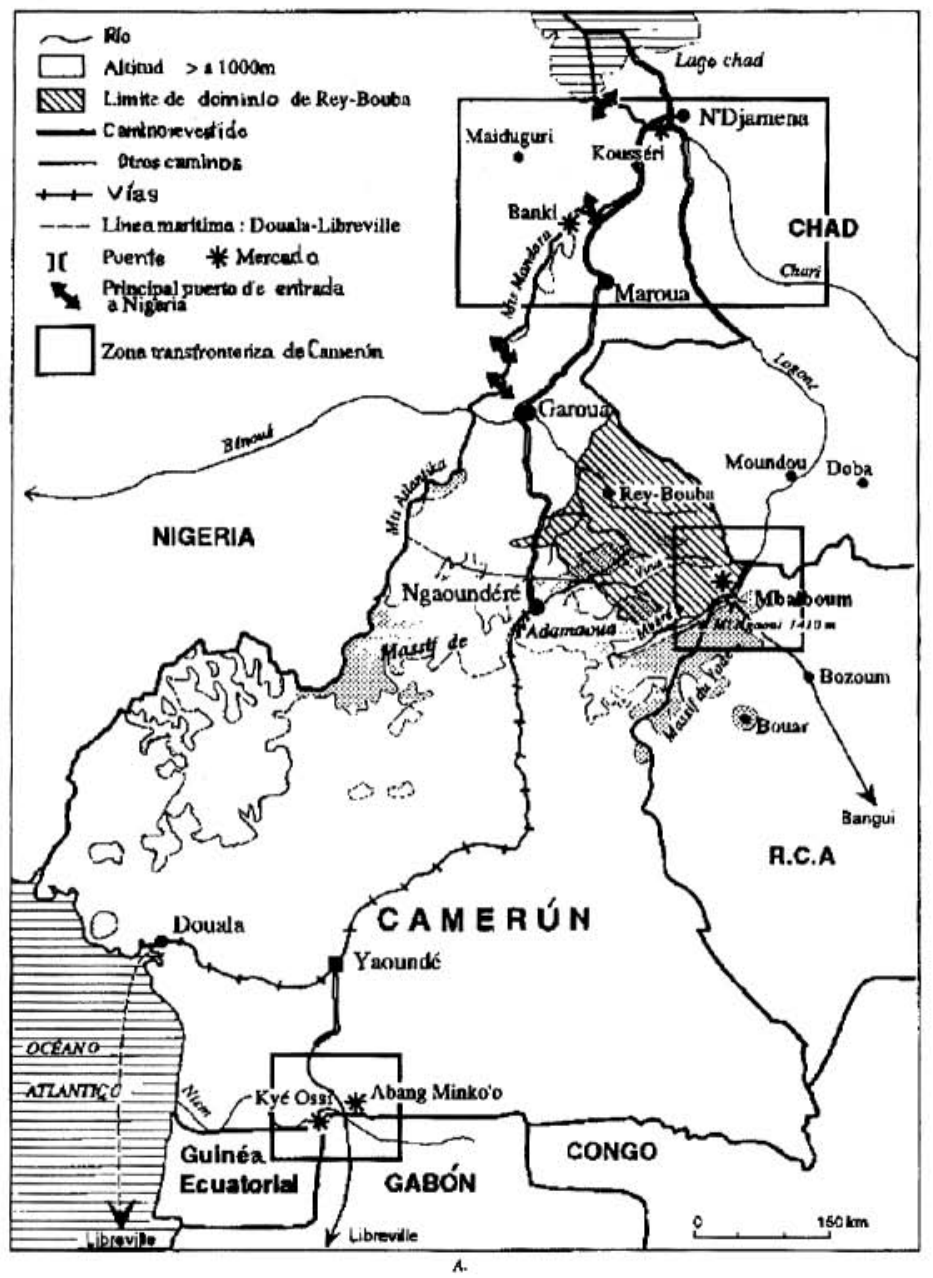

Figura 4. Ubicación de las zonas transfronterizas estudiadas en Camerún.

Fuente: Atlas Jeune Afrique de Camerún y de la RCA. 
tienen dificultades para penetrar en el mercado; están limitados a un lado del comercio particular, como la venta de las bebidas alcoholicas, actividad rechazada por sus compatriotas del norte por motivos religiosos. El caso del mercado nigeriano de Banki (en la frontera del extremo norte de Camerún (ver figura 4) es también elocuente. Aquí el comercio está dividido entre comerciantes Kanouris (originarios del norte de Nigeria), y son musulmanes y comerciantes ibos cristianos. Esas diferencias son a veces causa de violencia, cuando por motivos religiosos algunos disturbios sacuden el norte de Nigeria y encuentran repercusión en Banki: las tiendas de los ibos son entonces saqueadas. Otro ejemplo, el del mercado de Abang Minko'o (ver figura 4), situado cerca de la frontera entre Gabón y Camerún: ese mercado comerunés es marcado por una dualidad (y tensiones) de orden étnico enfrentando por una parte a los campesinos autóctonos Ntoumou (pertenecientes al grupo Fang), poco inclinados para comerciar, y por otra parte, a los comerciantes alógenos que vienen del oeste de Camerún (Bamikélé, Bamenda, Bamoun). Esas fronteras religiosas o éticas, y por lo general que tienen a la par fronteras lingüísticas (cada grupo tiene su dialecto), explican en parte las situaciones de enfrentamiento o las tensiones en el medio ambiente que se observan frecuentemente en los confines fronterizos. La frontera del Estado constituye un marcador de identidad tanto nacional como étnico.

\section{UNA EFERVESCENCIA DE ACTIVIDADES EN LAS FRONTERAS}

El punto común mayor de las fronteras de Estado estudiadas en África central es que todas son fronteras activas actualmente, animadas por un vaivén de personas y de mercancías más o menos intenso, según los lugares y los periodos. La frontera internacional se señala así por un papel de estímulos de los intercambios, papel al fin y al cabo reciente en ciertos casos (por ejemplo, desde los años ochenta entre Chad, RCA y Camerún y desde el principio de los años noventa entre Chad y Libia).

\section{La intensidad de los flujos de mercancías en las fronteras}

Sería escesivo calificar las transacciones comerciales transfronterizas como comercio informal. Diferentes tipos de transacciones operan, ya sean aparentes o subterráneas. El comercio aparente cubre a la vez el comercio oficial y el comercio paralelo. El primero es el practicado por sociedades industriales o comerciales declaradas y registradas en las diversas secretarías; sus resultados comerciales pueden ser calculados en cifras gracias 
a la existencia de un modo de gestión moderno. Las sociedades camerunesas Laking Textiles (venta de taparrabos) y CIMENCAM (cementera) son una tlustración. Los implantes fronterizos de los almacenes CIMENCAM recortan en ese tema el mapa de los mercados fronterizos cameruneses. Al lado de ese comercio oficial existe un comercio paralelo, también ostensible, pero que concierne a actividades privadas, individuales, con una estabilidad financiera variable. Tal comercio es difícilmente apreciable por la sencilla razón que tiene una contabilidad artesanal raramente rigurosa y tiene irregularidades más o menos grandes. Esas irregularidades van desde "arreglos" al fin y al cabo clásicos, con los aduaneros, hasta el contrabando que atañe productos prohibidos (gasolina nigeriana) o productos cuyos impuestos no han sido pagados. Al contrario de los tipos de transacciones anteriores, el comercio subterráneo concierne a operaciones cuya característica mayor es ser invisibles porque son ilícitos. Aquí no hay impuestos de mercancías o vitrinas, los intercambios se hacen de manera clandestina en la transtienda, lejos de las miradas. Los tráficos de oro, diamantes, drogas y armas (particularmente importante en la subregión) que solamente podemos intuir, constituyen indudablemente uno de los componentes comerciales de la mayoría de los mercados fronterizos de África central.

Los intercambios entre países de la zona UDEAC son dominados por las exportaciones camerunesas de productos industriales isualmente de consumo corriente hacia los países enclavados y subindustrializados (Chad, Centroafrica). Ciertos flujos intra-UDEAC están fundados sobre complementariedades ecológicas: Chad exporta productos del Sahel o sudano-sahelianos (productos y subproductos del ganado, natzón ${ }^{5}$, cebollas) y recibe bienes de la zona selvática como son la madera o café importados de Centroafrica.

La exportación de los productos de consumo local cameruneses (plátanos, plantainas, tubérculos, productos de huertos) con destino a Gabón, y en particular a Libreville, capital de Gabón, representa una corriente de intercambios mayor en el interior de la zona UDEAC: las potencialidades y los productos agrícolas son más o menos idénticos entre el Gabón selvático y el sur de Camenún (verdadero granero para el vecino Gabón), los flujos aquí no se deben a la explotación de cualquier diferencial ecológico sino a elecciones diferentes hechas por los dos países en materia de política y de inversión agrícola.

\footnotetext{
5 Carbonato de sodio recogido después de la evaporació: নel agua, en la región norte del lago Chad. El natzón es utilizado para la alimentación del ganado y los hombres, en particular para la preparación de salsas.
} 
El derrame de mercancías nigerianas (productos manufacturados de consumo corriente, carburo, carros y partes automotrices, materiales de construcción pero también medicinas y billetes falsos) hacia los países de la zona franca dan lugar a uno de los flujos más grandes en la subregión. El diferencial monetario (naira-franco CFA), la industrialización de Nigeria, la política nigeriana de subvención al consumo interno (para la gasolina), la herencia histórica de lazos culturales, políticos y comerciales (en particular en la región de lado Chad, dominada por redes mercantes Haoussa-Kanouri y Peul) figuran entre los principales determinantes de esos intercambios.

Entre los intercambios transfronterizos regionales, podemos también tener en cuenta las mercancías que vienen de Libia hacia Chad. La gama de esos arribos paralelos es amplia y comprende tanto productos manufacturados (partes automotrices, legía) como productos alimenticios (aceite, té verde) o gasolina. La mayoría de esos bienes son o productos libios (como la gasolina o las alfombras), o tienen como origen otros países árabes (Arabia Saudita, Marruecos, Tunes, Egipto) o países socialistas hermanos (China y Cuba). Ese comercio transfronterizo chado-libio afecta de manera desigual al territorio de Chad, el gradiente de difusión de la mercancías libias debilitado del norte al sur de Chad, a medida que aumenta la distancia respecto a la frontera libia. Si las regiones desérticas de la parte septentrional de Chad viven bajo el dominio comercial casi total de Libia (es el caso de Faya, la prefectura de Borkou-Ennedi-Tibesti), la capital de Chad, sin embargo, no se ve tan afectada. Sin embargo, los camiones de carga libios desde 1995 tienen la autorización de ir hasta la estación terminal de N'djamena y sus productos (sobre todo las pastas alimenticias) son ya más y más numerosos en los puestos de la capital. Ese tráfico terrestre meridiano entre Chad y Libia que reanuda con una época antigua (la del comercio en caravana precolonial a través de Sahara) se desarrolló desde el principio de la década de 1990 o luego de un cambio total de coyuntura política internacional: en primer lugar, la llegada en el poder en 1990 de Idriss Déby que fue seguida por la restauración oficial de las relaciones chado-libios y sobre todo, el arreglo de la controversia fronteriza de la banda de Aouzou (anexada en 1973 por Libia), al término del juicio hecho en 1994 por la corte internacional de La Haya en favor de Chad.

El caso de los intercambios chado-libios es así enriquecida con una enseñanza doble. Por una parte, confirma la influencia comercial y económica creciente de los operadores árabes y del medio oriente en África central, siguiendo la cancelación progresiva de sus compromisos (exceptuando en los sectores mineros y petroleros) hacia los protagonistas occidentales. La desclasificación geopolítica de la África negra a los ojos de 
los occidentales desde el final de los años ochenta tiene como consecuencias el desarrollo de nuevas influencias extranjeras, árabes, asiáticas (corea del sur) y de Europa oriental. Por otra parte, el ejemplo del comercio chado-libio pone a la luz la variabilidad del efecto-frontera en el tiempo. Herméticamente cerrada durante veinte años por la discrepancia de la banda de Aozou y de la guerra que deriva de ello), la frontera chado-libia fue primero mutilante, cuando estaba militarizada y defensiva; luego comunicadora y creadora de lazos en tiempo de paz y de cooperación. En esta perspectiva, revela muy bien el papel ambivalente de toda frontera. La variabilidad del efecto producido por la frontera se acentúa aún más a corto plazo como lo ilustran, por ejemplo, las clausuras ocasionales y repetidas de la frontera cameruno-nigeriana, por razones diplomáticas a menudo incidentes fronterizos en Bakassi. De la misma manera, la frontera cameruno-gabonesa es también objeto de clausuras puntuales, por lo general ligadas con la política de inmigración gabonesa particularmente restrictiva. Esas clausuras de fronteras constituyen pequeños eclipses temporales que ocasionan un agotamiento de los flujos en el espacio de algunos meses.

Fluctuando mucho en el tiempo, los intercambios transfronterizos se efectúan, para la gran mayoría, en mercados implantados cerca de la frontera. Esos mercados fronterizos a menudo periódicos (son animados durante 1, 2 ó 3 días a la semana) son centros de abastecimiento y de redistribución de mercancías. Juegan un papel clave en la estructuración de los espacios transfronterizos.

\section{La multiplicación de los mercados fronterizos}

Las zonas transfronterizas estudiadas tienen en común atraer uno o varios mercados, de los cuales muchos se han desarrollado desde hace una decena de años. El espacio Gabón-Camerún-Guinea Ecuatorial principalmente está centrado alrededor de otros mercados cameruneses de Abang Minko'o y de Kye Ossi, respectivamente, nacidos en 1992 y al principio de los años ochenta, el primero por la iniciativa del Estado, el segundo bajo el impulso de comerciantes privados.

La zona transfronteriza Camerún-Chad-RCA está estructurada por una jerarquía de mercados cameruneses, y dominada por el de Mbaiboum, desarrollado al principio de los años noventa. El mercado de Mbaiboum es relevado por pequeños mercados-satélites desparramados a lo largo de la frontera de Chad.

El espacio extremo norte de Camerún-nori de Nigeria-Chad se articula alrededor de un montón de mercados fronterizos en medio de los 
cuales figuran Benki (a horcajadas entre Nigeria y Camerún), Haiduguri (en el norte de Nigeria) y las ciudades gemelas de Kousseri (en Camerún) y D'djamena (ver figura 4). El mercado de Faya es uno de los puntos fuertes de la zona chado-libia.

Esos mercados difieren uno de otro por su historia y su génesis, más o menos antiguas, por su área de influencia, por su especialización más o menos adelantada y por su modo de gestión. El progreso fulgurante de Hbaiboum, aldea modesta de aproximadamente cien pescadores transformada en menos de 10 años en una plaza comercial de 15000 habitantes por la iniciativa de comerciantes privados, es difícilmente comparable con la historia de N'djamena. El área de atracción comercial de esos mercados fronterizos impacta por su extension: el mercado de Mbaiboum drena por ejemplo cada semana vendedores que vienen de Nigeria y del norte de Camerún, y clientes que llegan de Bangui (capital de centroafrica) pero también de Chad, de Sudán, del norte del Congo y del Zaire. De la misma manera el mercado de productos de consumo local de Abang Minko'o atrajo operadores económicos que tienen su base en Yaounde, Douala y Libreville. Esa atracción relativamente vasta de los mercados fronterizos hacen margenes fronterizos de los lugares de mezcla de poblaciones de diversas étnias y de diversas nacionalidades. Lejos de provocar un repliegue temeroso sobre sí mismo, la crisis en África fomenta la necesidad de apertura, como si el intercambio con el extranjero fuera una condición de sobrevivencia. El auge rápido del mercado de Mbaiboum también atrajo todo un contingente de oeste, africanos (senegaleses, gombianeses, ghaneanos, benineses), en general hombres jóvenes que están en tránsito y que acaparan durante su viaje pequeños oficios como son los portadores de agua, el zapatero ambulante, o el que jala un cochecito tipo chino. De allí el carácter particularmente abigarrado y cosmopólita de ese mercado que tiene parecido con la torre de Babel (los principales idiomas hablados son: el peul, el sango-hablado en RCA, el árabe, el pidgin-criollo inglés de Nigeria, el lingala —en vigor en el Congo- y el francés.

La mezcla que se efectúa en las fronteras se maneja a veces en un clima de serenidad relativa entre comunidades (en Kyé Ossi, en Mbaiboum), y a veces en un clima de tensión. Lo vivido de la frontera es así particularmente difícil para los cameruneses y los ecuato-guineanos que vinieron a trabajar a Bitán, pequeña ciudad fronteriza del norte de Gabón (ver figura 4): las discriminaciones, la vejaciones y las violencias que padecen los extranjeros por parte de los gaboneses crean por un efecto lógico de reacción, una cierta solidaridad entre nacionales del mismo país, aun entre extranjeros. En la frontera aumentan a menudo los sentimientos de pertenencia nacional. Sin embargo, hay ciertos matices porque lo vivido en 
la frontera ya no es lo mismo si uno es miembro de la étnia local (cuya área de población está encima de la frontera) o bien si uno es originario de una región lejana. La primera situación permite un juego sobre la frontera y la alternancia de nacionalidades: algunos Fangs del sur de Camerún se identifican simultáneamente como cameruneses, ecuato-guineano o gaboneses según las circunstancias y las autoridades que se encuentren. Igualmente, un comerciante del norte de Camerún que habla perfectamente bien el Haoussa y el pidgin (hablados en el norte de Nigeria) puede ocuparse de sus asuntos en el país vecino sin inquietarse por la condición de obtener una etiqueta de impuestos (que comprueba que pagó sus impuestos nigerianos) y de vestirse con la moda nigeriana. La frontera engendra esas situaciones insólitas, tal es la de ese comerciante camerunés de Banki, Kanouri originario del Estado de Borno en Nigeria, y que posee dos tiendas en el mercado, una del lado camerunés, y la otra del lado nigeriano (la frontera atraviesa el mercado de Banki en su centro, en medio de los puestos y de las casas). Que decir de esos dos individuoshijos de los mismos padres, uno es camerunés y el otro gabonés.

La confusión es aún más fuerte en el caso de matrimonios mixtos. Si la mención de la nacionalidad aparece como una distinción ficticia y confusa para individuos de la misma familia o de la misma étnia, separados por la frontera, es una diferenciación que la población sabe usar cuando se requiere, atestiguando así de la relación circunstancial y utilitaria que mantienen con la frontera.

La preponderancia de las implantaciones de los más importantes mercados fronterizos del lado nigeriano y camerunés en África central es uno de los fenómenos notables. Parecería que cada mercado fronterizo tiende a implantarse del lado del país vendedor, proveedor de mercancías que tiene que despachar, lo que se confirma bien con el Camerún y Nigeria. Los tenderos tienen en general menos dificultades para instalarse en su propio país que en territorio extranjero. Así pues, en Mbaiboum, en Abang Miko'o, los controles de los aduaneros y policías cameruneses para los extranjeros son netamente menos puntillosos que los de sus homólogos del Chad, de Centroafrica o de Gabón. Por otra parte, los problemas políticos interiores del Chad (violencia) y de centroafrica (motines, tentativas de golpe de Estado) no estimulan la implantación de comerciantes. La política comercial incitativa del Estado camerunés que combina la creación de mercados fronterizos, la práctica de impuestos moderados sobre las mercancías y la tolerancia de una libre circulación de los extranjeros hacia los mercados fronterizos cameruneses contrasta con la política blanda de los Estados gaboneses y centroafricanos en fave de la promoción de los espacios fronterizos. 
El estudio de los principales mercados fronterizos de África central revela la existencia de una misma convergencia de factores que presiden al desarrollo de un mercado. El factor clave necesario para el éxito de un mercado fronterizo es la presencia de un servicio de comunicación carretero que tiene la calidad indispensable para permitir el tránsito de camiones de carga hacia los centros de abastecimiento y de consumo del interior del país. Es el revestimiento con asfalto del tramo nigeriano Baha-Banki y la reparación de la carretera camerunesa Mora-Limani que aseguraron el auge del mercado de Banki. Igualmente, Mbaiboum debe su apertura a la construcción, por parte de la RCA de un sólido puente, frontera que pasa encima del río Mbere (los centroafricanos son los principales clientes) y sobre todo a la rehabilitación y al mantenimiento por parte de la SODECOTON ${ }^{6}$ de un camino de terracería rural regional que abre el acceso a la frontera oriental camerunesa desde los caminos interiores de Garoua y de Ngaoundere (ver figura 4).

Los otros elementos indispensables para la apertura de un mercado fronterizo son la existencia de una renta de situación geográfica, a menudo una situación de crucero de primer orden (Mbaiboum y Kye Ossi se sitúan por ejemplo en la intersección de tres fronteras), un diferencial económico de gran tamaño de ambos lados de las fronteras (oponiendo en particular áreas consumidoras y áreas proveedoras de mercancías), una circulación monetaria relativamente intensa en la región fronteriza que puede ser relacionada con una cultura comercial como el algodón hacia Mbaiboum o la cocoa y los productos de consumo local hacia Kye Ossi. Son igualmente requeridos un relajamiento de la soberanía del Estado sobre esos confines y un mínimo de densidad humana regional (al menos 20 $\mathrm{hab} / \mathrm{km}^{2}$ ). La ausencia del mercado fronterizo a proximidad inmediata de la frontera chado-libia es quizás debido al carácter demasiado tenue de la población: los mercados fronterizos deben de coincidir con las ciudadesoasis como Faya, que a veces están situadas a varias centenas de kilómetros de la frontera pero que ofrece una concentración humana mínima.

Con todos esos factores, puede entonces emerger un espacio de crecimiento periférico, estructurado con uno o varios mercados ¿pero, esa zona transfronteriza naciente puede inducir afectos duraderos en el plan espacial, político y socioeconómico?

6 La SODECoton es la Sociedad Camerunesa de Desarrollo del cultivo del Algodón, una sociedad de Estado con capitales mixtos y de la cual la compañía francesa de desarrollo de los textiles es accionaria. 


\section{ICI. IMPACTO POLÍTICO Y TERRITORIAL DE LAS ZONAS TRANSFRONTERIZAS}

\section{Regiones axiales, a la configuración movediza}

Las regiones transfronterizas de África central difícilmente pueden ser tomadas como espacios concéntricos homogéneos, estrictamente conscriptos por contornos lineales, delimitados en el interior de los territorios nacionales. Se parecen más bien a espacios lineales formados por gradaciones sucesivas y estructurados en función de ejes y de puentes (terrestres, marítimos y aéreos), que están ellos mismos flanqueados y dinamizados de mercados fronterizos con un papel de pivote.

Se desdibujan así, espacios distendidos, estirados a lo largo de las carreteras, de la línea aérea o marítima. El espacio fronterizo chado-libio, reducido a carreteras transaharianas de orientación meridiana, es caricaturesco de ese tipo de región axial. Las fuertes molestias naturales (el desierto) y la herencia pesada de los puntos litigiosos de la banda de Aozou (que llega hasta un suelo minado) se conjugan aquí para restringir la población e impedir un desarrollo transfronterizo en forma de areola.

Una de las zonas transfronterizas más conplejas es sin duda la zona que está en el extremo norte de Camerún, el norte de Nigeria y el Chad. La multiplicidad de las fronteras (fronteras estatales, frontera entre zona franca y naira, frontera UDEAC, aeropuerto internacional de N'djamena) acrecenta la dificultad para comprender los límites. Densamente poblada, esa nebulosa se estructura a la vez en función de un florecimiento de flujos y de intercambios a distancia (por ejemplo, Lagos-Maiduguri-N'djamena) y en función de un florecimiento de flujos de corto alcance (Maroua-Banki, N'djamena-Kousseri) que segmenta fuertemente el espacio: Maiduguri, Maroua, N'djamena constituyen los principales núcleos de ese conjunto fragmentado, polinuclear, y los centros de estallamiento hacia destinos más lejanos. La densidad de la población, la multitud de los mercados fronterizos, la intensidad de los desplazamientos, la preponderancia casi exclusiva del comercio en las actividades regionales convergen en hacer de la región una de las zonas transfronterizas de las más terminadas.

De más fácil acceso, los espacios transfronterizos Camerún-GabónGuinea Ecuatorial y Camerún-Chad-Centroafrica presentan analogías en cuanto a su estructuración interna: zonas "digitadas" organizadas por flujos transversales lejanos que tienen ejes que son fácilmente identificados (por ejemplo: eje marítimo Libreville Douala, eje carretero Libreville-Oyem-Yaounde vía Kye Ossi y Asang Miko'o en el primer caso, eje Mbaiboum-Ngaoundere, Mbaiboum-Garoua, Mbaiboum-Bangui, en el 
segundo). Aquí, los puntos fuertes de la zona transfronteriza son fácilmente identificados porque están menos diseminados y son menos numerosos que en el ejemplo anterior: solo uno o dos mercados fronterizos atraen la zona transfronteriza, resaltando de manera muy visible de la zona de los alrededores.

Espacios lineales y con espacios vacíos, los espacios transfronterizos son señalados también por una organización espacial extremadamente movediza como lo muestra la expansión errática de los mercados y la fluctuación de los itinerarios tomados por los flujos. Los ejes de transporte que llevan a las fronteras no dejan de desplazarse según lo práctico de las carreteras, las variaciones locales de los controles policiacos, la evolución de los precios de transporte, la aparición imprevista de nuevas rutas de carga, etcétera. Testigo de esa perpetua recomposición de las dinámicas fronterizas es el fantástico cabotaje en vigor desde 1993 entre los puertos de Douala y de Libreville, que constituye una gigantesca captura de flujos (esencialmente de los productos de consumo local que son llevados de Camerún hacia Libreville, y que se efectúa por la vía marítima más que por vía terrestre. Esa modificación de itinerario es imputable aquí a la descompostura de la panga que permitía a los camiones atravesar el río-frontera (el NTEM) entre Camerún y Gabón (ver figura 4).

Esa fugacidad característica del funcionamiento de las zonas transfronterizas se debe a su quinta esencia misma, la de los espacios-movimientos (Braudel) alimentados de fluidez y cuya dinámica viene totalmente del sistema de circulación, que se detiene después de un hundimiento de un puente, de la quebradura de una panga, de una carretera mal conservada o destruida, de una multiplicación de barreras carreteras o de un crecimiento de la inseguridad que provoca que el ánimo decaiga, el mercado fronterizo zozobre, y los comerciantes se alejen. Los ejemplos abundan de antiguas plazas comerciales antes atractivas y hoy desaparecidas, a ejemplo de Ngaoui (eliminada por Mbaiboum en la frontera de Camerún y de RCA), Kerawa (ahogada por Banki en la frontera Camerún-Nigeria) Ababg Miko'o (suplantada por Kye Ossi en la frontera de Gabón y de Guinea Ecuatorial).

Porque mañana pueden aparecer en otra parte nuevas polarizaciones como consecuencia de una carretera rehabilitada o después de un refuerzo o de los controles policiacos, pero también porque sacan su dinámica de la única actividad, la comercial, (lo que les hace particularmente vulnerables en caso de un cambio total de conyuntura). Las regiones transfronterizas son, ante todo, costrucciones provisionales. Por ese hecho, difícilmente se pueden considerar como lugar de un desarrollo futuro o como lugares susceptibles de perjudicar la integridad del territorio nacional. 


\section{A promoción de los espacios transfronterizos conforta paradójicamente ul listado y el marco territorial antiguo}

Puryue su funcionamiento está colocado bajo el signo de lo efímero, las munis transfronterizas no parecen tener la capacidad de producir efectos duraderos. El examen de las inversiones inmobiliarias operadas sobre los márgenes fronterizos es en ese aspecto muy revelador.

En todos los casos estudiados, las inversiones efectuadas por los comerciantes sobre el sitio mismo de la frontera o en el nivel del mercado Ironterizo son limitadas, con la excepción de N'djamena, que por el hecho Je ser capital, capta una parte de las inversiones de los comerciantes (construcción de casa, de almacenes en duro [ladrillo], de mezquite, renta Inınobiliaria, creación de sociedad de transporte). En otras partes, en el resto de la zona Nigeria, Camerún, Chad, son sobre todo ciudades interiores (Maroua, Garoua, Maiduguri) que concentran las inversiones. Es verdad también que los grandes comerciantes que pueden volver a invertir rara vez eligen una ciudad fronteriza. La pequeña ciudad de Banki es una excepción en la medida que varias grandes familias de comerciantes originarios de Borno viven allí. Esas personas de renombre locales han hecho construir mansiones, varios depósitos y almacenes en duro, así como una mezquita.

Pero a menudo, las únicas construcciones sólidas que se encuentran en el lugar mismo de la frontera son infraestructura socioeducativa (escuelascentro de salud) o religiosa. La construcción de mansiones es escasa, a veces a causa de las autoridades locales; por ejemplo, la alcaldía de Olamze prohibe las construcciones sólidas sobre el mercado de Kye Ossi, argumentando razones estratégicas, el peligro que representa la proximidad del vecino ecuato-guineano. Igual en Mbaiboum, el poder de prebenda del jefe Peul ordena evitar mostrar cualquier señal exterior de riqueza bajo la pena de ser más gravado, de allí, la fachada de una cartolandia de ese mercado, compuesto de casas construidas de barro o con sencillos materiales de origen vegetal. La inseguridad relacionada al fenómeno de los cortadores de carretera ${ }^{7}$, que afecta muchos confines territoriales en África central, no estimula tampoco las inversiones. En total, la precariedad de las construcciones es común sobre los mercados fronterizos porque las inversiones de los operadores económicos se efectúan prioritariamente en los centros urbanos del interior dotados de una permanencia segura. Los comerciantes viven frecuentemente divididos entre dos residencias, una

7 Así se les llama a los ladrones que atacan y roban en bandas armadas, a los comerciantes. 
en la frontera, y la otra en una ciudad más alejada del interior, que cobija en general a mujeres y niños.

El estudio de las inversiones permite entrever el papel clave de las capitales provinciales y de las ciudades secundarias en la organización del espacio transfronterizo. Bien comunicadas con la capital o el gran centro económico portuario (a menudo por vía aérea), contituyen la articulación indispensable entre, por una parte, el puerto o la capital, y por otra parte, el mercado fronterizo, que se sitúa a menudo en el campo. Es en esas ciudades medianas que a menudo son devueltos a la patria, por lo menos en un primer tiempo, los beneficios en líquido realizados el o los días de mercado con el fin de ponerlos a salvo en bancos o cajas fuertes particulares. Los mercados fronterizos también dependen de los centros urbanos interiores para el abastecimiento y el consumo de las mercancías. Aquéllos pues deben de ser incluidos en las redes tranfronterizas de las cuales constituyen las extremidades.

Así se ve cuan erróneo es considerar o hablar de una cierta autonomía de las zonas transfronterizas, en primer lugar, porque dependen de los proveedores y de clientes que tienen su base en ciudades interiores, a veces muy alejadas; en segundo lugar, porque necesitan infraestructura nacional de transporte, aunque sea para el transporte y la distribución de las mercancías (éstas son escasamente producidas en el lugar). Cierto, las zonas fronterizas comercialmente dirigidas hacia el país vecino para su abastecimiento (pensamos en la ciudad de Faya que mira hacia Libia) ofrecen un panorama que no es favorable a la consolidación del rompecabeza territorial nacional. Sin embargo, sería exagerado y apresurado ver allí un peligro de fragmentación territorial, ninguna tendencia secesionista se ha manifestado políticamente, menos aún cualquier deseo por parte de la población de adherirse a Libia.

De manera general, parece pues que la agitación transfronteriza tenga paradojicamente efectos centrípetas: tiene lugar un efecto únicamente gracias a un desenclave de los márgenes por las infraestructuras de transportes (en general, una carretera) que contribuyan, más o menos en solidificar las periferias fronterizas con el centro del territorio: el norte gabonés (lindando los mercados de Kye Ossi y de Abang Minko'o), el noroeste centroafricano (fronterizo de Mbaiboum) o el noreste de Camerún todos han sido objeto de una política nacional de mejoramiento de los servicios de comunicación carreteros.

Ese tipo de aserción se verifica sin embargo mucho menos cuando las zonas fronterizas ocupan una posición central: es el caso de la región de $\mathrm{N}$ 'djamena, capital fuera de su centro, situada a la orilla de la frontera, pero que constituye el centro del país, en término de mando y de poder. 


\section{CONCLUSIÓN}

La emergencia de las zonas transfronterizas, basadas en el comercio, que se puede observar desde hace diez años en África central forma un nuevo modo de regulación socioeconómica vital para países que se encuentran en una situación de callejón sin salida económico y político. Polarizadas por mercados y estructuradas en función de ejes de comunicación, esas zonas transfronterizas son espacios de redecillas y distendidos cuya organización interna es muy cambiante. Esos espacios están completamente articulados con los centros interiores del territorio sin los cuales no pueden funcionar. Lejos de encarnar un regionalismo transestatal con un dinamismo depredador y desintegrador para los Estados en cuestión, la efervescencia fronteriza contribuye, por el contrario, en asegurar la perenidad del Estado y el mantenimiento del recorte territorial antiguo. En esa perspectiva, los espacios fronterizos son un laboratorio de primer orden para estudiar el Estado y observar el "aggiornamento" de las modalidades del ejercicio del porder ¿No sería sin embargo que se aprende sobre los márgenes lo que es el centro?

\section{BIBIOGRAFÍA}

BAYARD, J.F. 1989. L'Etat en Afrique, París, Fayard, 440 p.

BAYARD, J.F. Ellis, S. y Hibou, B. 1997. La criminalisation de l'Etat en Afrique, Bruxelles, ed. Complexe, $167 \mathrm{p}$.

FOUCHER, M. 1991. Fronts et frontières. Un tour du monde gèopolitique, París, Fayard, 691 p.

EGG, J. y Igue, J.O. 1993. L'intégration par les marchés dans le sousespace est: I' impact du Nigeria sur ses voisins immédiats. Rapport de synthèse, INRA-IRAM-UNB, Club du Sahel/OCDE/CILSS.

LABAZEE, P. 1995. "Dynamismes entreneuriauz et intégration régionale par le bas en Afrique au Sud su Sahara”, communicarion au colloque international de Mexico sur la compétitivité territoriale et recomposition socio-politique, $25 \mathrm{p}$.

RAISON, J.P. 1993. "Les formes spatiales de l'incertitude en Afrique contemporaine" en Espaces africains en crise, Travaux géographique de Reims, núm. 83-84:5-18. 